\title{
A study of electromechanical switching in ferroelectric single crystals
}

\author{
J.H. Yen ${ }^{\mathrm{a}}$, Y.C. Shu ${ }^{\mathrm{a}, *}$, J. Shieh ${ }^{\mathrm{b}}$, J.H. Yeh ${ }^{\mathrm{b}}$ \\ anstitute of Applied Mechanics, National Taiwan University, Taipei 106, Taiwan, ROC \\ ${ }^{\mathrm{b}}$ Department of Materials Science and Engineering, National Taiwan University, Taipei 106, Taiwan, ROC
}

Received 8 August 2007; received in revised form 14 February 2008; accepted 15 February 2008

\begin{abstract}
This article documents both modeling and experimental studies developed to investigate the switching behavior of ferroelectric single crystals. The theoretical model makes a priori ansatz that switching follows the evolution of a particular domain pattern. The choice of this configuration is dictated by the requirement that domains remain compatible during evolution, giving rise to a low-energy path for the overall switching. The construction of this pattern is achieved using multirank laminates. It offers an advantage of specifying different types of domain wall movements, leading to a distinction for the switching types. A loading experiment is performed on a barium titanate $\left(\mathrm{BaTiO}_{3}\right)$ single crystal with a constant compressive stress and a cyclic electric field. Both $180^{\circ}$ and $90^{\circ}$ coercive fields are measured as input parameters required for the theoretical framework. The simulation results show good agreement with the observed strains measured by the present and other available experiments. It is found that depolarization has a non-trivial influence on attainable actuation strains.
\end{abstract}

(C) 2008 Elsevier Ltd. All rights reserved.

Keywords: Compatibility; Depolarization; Domain switching; Electromechanical processes; Ferroelectric single crystals

\section{Introduction}

Ferroelectric materials exhibit highly characteristic electromechanical coupling with the ability to switch polarization in response to an external electric field or mechanical load. They are piezoelectric under poling and are often used in various actuator and sensor applications due to high-frequency response and low hysteresis (Uchino, 1998; Shieh et al., 2001; Bhattacharya and Ravichandran, 2003). However, the strains that they display under piezoelectric response are typically small. This motivates numerous experimental efforts using ferroelectric single crystals to obtain large actuation strains. It can be achieved either by enhancing their intrinsic contribution through composition control and material development (Park and Shrout, 1997), or by using their extrinsic contribution through domain switching (Burcsu et al., 2000, 2004; Ren, 2004; Cao, 2005).

\footnotetext{
*Corresponding author. Tel.: + 886233665627 ; fax: + 886233665628.

E-mail address: yichung@spring.iam.ntu.edu.tw (Y.C. Shu).
} 
This article focuses on the latter approach and combines both modeling and experimental studies aiming at understanding the fundamental process of ferroelectric switching in single crystals.

Ferroelectric crystals are phase-transforming materials. There exists a critical temperature, called the Curie temperature, such that above this temperature they are paraelectric with non-polar structures. However, on cooling below the Curie point, the unit cell is spontaneously polarized with a spontaneous lattice distortion. In this state, the crystals are both piezoelectric and ferroelectric where the piezoelectric behavior exhibits along the spontaneous polarization direction. Another feature of this transformation is that there is a reduction in crystallographic symmetry, and this creates more than one symmetry-related polarized state. Each of them is equally favorable in the absence of external field and load. These preferred states, which are characterized by pairs of spontaneous strain and polarization, are commonly called the ferroelectric variants. Very often, a mixture of variants rather than a single variant coexists in these materials; and multiple domains, each of which is a region of uniform spontaneous strain and polarization, are observed with scales ranging from few to hundreds of nanometers. Adjacent domains are separated by a domain wall which has a well-defined crystallographic orientation. As a result, domains form highly intricate and characteristic patterns in ferroelectric materials (Arlt and Sasko, 1980; Wada et al., 1999; Ricote et al., 2000). Besides, the type of a domain wall is determined by the angle between the polarizations on the two sides of the wall, leading to $180^{\circ}$ and non- $180^{\circ}$ walls.

Domains can be switched from one variant to another under an electric field or stress since the variants have equal energy. While the energy barrier for the homogeneous switching of two different variants is high, the switching can start with a nucleation of the domain wall and proceeds by the propagation of wall (Little, 1955; Loge and Suo, 1996; Hong et al., 1999). As a domain wall sweeps through a crystal, one domain expands at the expense of the other, leading to the conversion of one domain type to another. Traditionally switchings are classified according to the types of walls. A $180^{\circ}$ switching reverses polarization only and has no effect on shape change since variants on the two sides of a $180^{\circ}$ wall have an identical crystal orientation. However, a non- $180^{\circ}$ switching is accompanied with a significant change in shape due to different crystallographic orientations. Both switchings can be driven by suitable electric fields, but stress can only induce a non- $180^{\circ}$ domain switching. Combining all these features suggests that a large actuation strain can be achieved through non- $180^{\circ}$ domain switching under a suitable electromechanical loading.

The key behind this idea is that walls separating different variants cannot be arbitrary; instead, they have well-defined crystallographic orientations as mentioned above. A wall with different strains and polarizations on its two sides must be oriented in a particular way to remain connected and uncharged. In other words, its orientation is determined to guarantee that strain and polarization are compatible across the wall. Violation of this rule results in additional stress and depolarization field, and hence, increasing the energy of the material. Thus, it suggests that compatible domain walls provide a low-energy path for domain switching, and the understanding of them leads to novel strategies of large strain actuation. Indeed, Shu and Bhattacharya (2001) have adopted an energetic argument to systematically exploit various types of walls for polarized tetragonal, rhombohedral and orthorhomibic ferroelectric crystals. Following their analysis, Burcsu et al. (2004) have employed a combined electromechanical loading to induce $90^{\circ}$ switching in $\mathrm{BaTiO}_{3}$ single crystals, and a strain of $0.8 \%$ is measured at $1.78 \mathrm{MPa}$ compressive stress together with a moderate cyclic electric field. While this level of strain is several times larger than that induced by piezoelectric response, it is smaller than the theoretical maximum $1.09 \%$ for $\mathrm{BaTiO}_{3}$ single crystals (Shu and Bhattacharya, 2001). Further, although measured with an entirely different loading setup to the previous one, a significant strain reduction to $0.45 \%$ is also observed in the present study (see Section 3 for a complete description). Various reasons are proposed; the common interpretation for the strain reduction is due to coercive hardening introduced in many phenomenological switching models (Kamlah and Tsakmakis, 1999; McMeeking and Landis, 2002; Landis, 2002). Recently, Shilo et al. (2007) have proposed another mechanism to explain it. They have assumed the existence of friction between the ferroelectric crystal and the mechanical loading device of Burcsu et al.'s experiment, showing that the strain is reduced due to this factor. As the hardening in coercive fields is typically small for single crystals (Cao, 2005) and the present experiment is conducted in an oil bath, the effects of coercive hardening and friction could be negligible in the current case. Therefore, other possible mechanisms causing the strain reduction on switching need to be burrowed out and are investigated in this article. 
On modeling the nonlinear switching behavior of ferroelectric materials, various frameworks based on continuum descriptions have been proposed. They can be grouped into three major categories: phase-field models (Chen, 2002; Zhang and Bhattacharya 2005a, b; Dayal and Bhattacharya, 2007; Shu and Yen, 2007; Shu et al., 2008), phenomenological constitutive models (Bassiouny et al., 1988a, b; Kamlah, 2001; Landis, 2004) and micromechanical constitutive models (Hwang et al., 1995; Lu et al., 1999; Kessler and Balke, 2001; Huber et al., 1999; Huber, 2005). The phase-field approaches typically are computationally expensive and impractical for applications, and the phenomenological models are usually established to describe the ferroelectric switching in polycrystals based on a reduced set of fitting parameters. Both are beneficial in different concerns, but may not be suitable to describe the switching behavior of single crystals. Instead, micromechanical models are developed directly with the description of domain switching at the crystal lattice scale as the motivation. They differ in the way of specifying switching criterions (Sun and Achuthan, 2004; Zhang et al., 2006). Many of them start with the constitutive construction in the level of a single crystal, and model the polycrystalline behavior by appropriate averaging of the grain responses using either the Reuss approximation (Hwang et al., 1995; Lu et al., 1999), the self-consistent average scheme (Chen et al., 1997; Huber et al., 1999; Landis and McMeeking, 2001), or the finite element method (Fotinich and Carman, 2000; Kim and Jiang, 2002; Kamlah et al., 2005). However, early works of this approach have treated the single crystal behavior in an oversimplified manner by assuming domain switching is a sudden change from one variant to another (Hwang et al., 1995; Chen et al., 1997; Michelitsch and Kreher, 1998). A significant improvement is made by Huber et al. (1999) who introduce the incremental switching in analogous to the operation of a slip system in crystal plasticity. It includes a kinematic description of the remanent strain and remanent polarization, a switching criterion, and a hardening law for single crystals. Experimental verifications of this model have been provided by Huber and Fleck (2001) and Shieh et al. (2003). Other modified micromechanical models for ferroelectric single crystals include the work by Elhadrouz et al. (2005) who introduce an interface operator to account for interaction energy, and the work by Liu and Lynch (2003) who investigate relaxor crystals poled along different crystallographic directions. Finally, Seelecke et al. (2005) and Kim and Seelecke (2007) have proposed different frameworks for switching in ferroelectric single crystals. They generalize the one-dimensional free energy model by Smith et al. (2005) to two- and three-dimensional cases based on the theory of thermally activated processes and statistical thermodynamics.

The present approach is motivated by several recent works such as Li and Weng (2002, 2004), Rödel and Kreher (2000, 2003), Fulton and Gao (2001) and Landis and McMeeking (2001). Different from most of switching models in the literature, they include geometric arrangements of domains within either single crystals or individual grains, and typically lamellar domains are assumed in their models. Nevertheless, only a subset of all possible variants is taken into account and some of the domain arrangements may not be compatible structures. This calls for a detailed analysis of microstructural evolution and the relevant coupling effects. Indeed, the switching model proposed here assumes that switching is enabled by the evolution of a particular domain pattern. The choice of this domain configuration is motivated by the fact that it is consistent with the applied boundary conditions in the sense that it accommodates the overall prescribed strain and polarization, and that it minimizes the energy including the non-local electrostatic contribution. The construction of this domain pattern is achieved using multirank laminates ( $\mathrm{Li}$ and Liu, 2004). As a result, domains remain compatible during evolution and the different types of domain wall movements can be specified explicitly. The former guarantees that the overall switching follows a low-energy path, and the latter distinguishes the switching types such as $180^{\circ}$ and $90^{\circ}$ switchings for tetragonal ferroelectric crystals. Another feature of the proposed framework is that the depolarization energy arising from the local polarizations in the crystal is taken into account. This effect is often neglected in numerous switching models, while it may be important to explain switching hardening in strain and certain abnormal behavior observed in recent experiments on $\mathrm{BaTiO}_{3}$ single crystals. The former has been briefly examined by Shu et al. (2007) and the latter has been reported and discussed by Shieh et al. (2007).

This article starts with a description of electroelastic potential energy of a ferroelectric crystal proposed by Shu and Bhattacharya (2001) in Section 2. Common lamellar and banded patterns are illustrated and are extended to electromechanically compatible multirank laminates. Driving forces are derived by the reduction of potential energy, and dissipations are determined in terms of different kinds of domain wall movements. 
With the balance of these two forces, the switching problem is investigated in the case of flat-plate configuration and extended to other cases with complicated configurations. Section 2 is ended with an interesting comparison between the present and Huber et al.'s switching models at the level of single crystals (Huber et al., 1999). Substantial differences between these two are found in the kinematic descriptions of switching variants and in the expressions of driving and resistance forces. After that, an experiment to investigate the electrostrictive behavior of a $\mathrm{BaTiO}_{3}$ single crystal under the flat-plate configuration is described in Section 3. Both $180^{\circ}$ and $90^{\circ}$ coercive fields are measured as input parameters required for the theoretical framework. The simulation results taking into account the depolarization effect are offered in Section 4. They are compared to the observed strains measured by the present and Burcsu et al.'s experiments (Burcsu et al., 2004). Finally, conclusions are drawn in Section 5.

\section{Framework}

\subsection{Electroelastic energy of a ferroelectric single crystal}

Consider a ferroelectric single crystal occupying the region $\Omega$ at a fixed temperature. The state variables describing the electromechanical behavior of it are displacement $\mathbf{u}$ and polarization $\mathbf{p}$. As the relative changes of spontaneous distortion from the paraelectric to ferroelectric state are typically around the order of $10^{-3}$ for most perovskite ferroelectrics, the linearized symmetric strain is assumed here. It is denoted by $\varepsilon$ and is related with displacement by $\varepsilon[\mathbf{u}]=\frac{1}{2}\left\{\nabla \mathbf{u}+(\nabla \mathbf{u})^{\mathrm{T}}\right\}$.

Suppose the crystal is under a combined electromechanical loading. Let $\mathbf{E}^{*}$ be the applied electric field generated in the absence of ferroelectric crystal, and $\boldsymbol{\sigma}^{*}$ be the symmetric stress induced owing to the applied traction on part of its boundary. Notice that $\sigma^{*}$ has to be divergence-free in the absence of body forces and to be consistent with the mechanical boundary conditions (Shu et al., 2004). Shu and Bhattacharya (2001) have proposed that the state variables $\mathbf{u}$ and $\mathbf{p}$ of a ferroelectric crystal can be obtained by minimizing its electroelastic energy given by

$$
I(\mathbf{u}, \mathbf{p})=\int_{\Omega}\left\{W(\varepsilon[\mathbf{u}], \mathbf{p})-\mathbf{E}^{*} \cdot \mathbf{p}-\boldsymbol{\sigma}^{*} \cdot \varepsilon[\mathbf{u}]\right\} \mathrm{d} \mathbf{x}+\frac{\varepsilon_{0}}{2} \int_{\mathbb{R}^{3}}|\nabla \phi|^{2} \mathrm{~d} \mathbf{x},
$$

where $W$ is the stored energy density of the crystal depending on strain and polarization, $\varepsilon_{0}=8.85 \times$ $10^{-12} \mathrm{C}^{2} \mathrm{~N}^{-1} \mathrm{~m}^{-2}$ is the permittivity of free space, and the electric potential $\phi$ is obtained by solving the Maxwell's equation

$$
\nabla \cdot\left(-\varepsilon_{0} \nabla \phi+\mathbf{p} \chi_{\Omega}\right)=\rho_{\mathrm{f}} \quad \text { on } \mathbb{R}^{3}
$$

subject to some appropriate electrical boundary conditions. The domain wall energy in Eq. (1) is neglected due to the consideration of a bulk specimen (DeSimone, 1993). Further, in Eq. (2), $\rho_{\mathrm{f}}$ is the free charge density and $\chi_{\Omega}$ is the characteristic function of $\Omega$ such that $\chi_{\Omega}(\mathbf{x})=1$ if $\mathbf{x} \in \Omega$ and $\chi_{\Omega}(\mathbf{x})=0$ otherwise.

The physical interpretation of each term in Eq. (1) is given here. The first term, the stored energy density, accounts for the energetic cost that the crystal must pay if the strain and polarization deviate from the preferred states. Thus it contains the information that the crystal prefers certain spontaneous strain and spontaneous polarization. The second and the third terms are the potential energies due to the applied electric field and the mechanical load, and thus, this enforces the desire of the vectorial direction of polarization as well as the tensorial direction of strain to align with the applied field and load. The second integral in Eq. (1), called the depolarization energy, is the electrostatic self-energy associated with the electric field generated by the spontaneous polarization of the crystal itself.

The properties of the stored energy density $W(\boldsymbol{\varepsilon}, \mathbf{p})$ must reflect the fact that the reduction in crystal symmetry from the paraelectric to ferroelectric phase implies that the ferroelectric crystal has several ground states (variants) in the strain-polarization space. Indeed, below the Curie temperature the crystal is spontaneously polarized with a spontaneous strain. Let $N$ be the total number of variants denoted by $\left(\boldsymbol{\varepsilon}^{(i)}, \mathbf{p}^{(i)}\right)$ for $i=1,2, \ldots, N$. They correspond to the energetic ground states of $W(\boldsymbol{\varepsilon}, \mathbf{p})$ such that at any position 


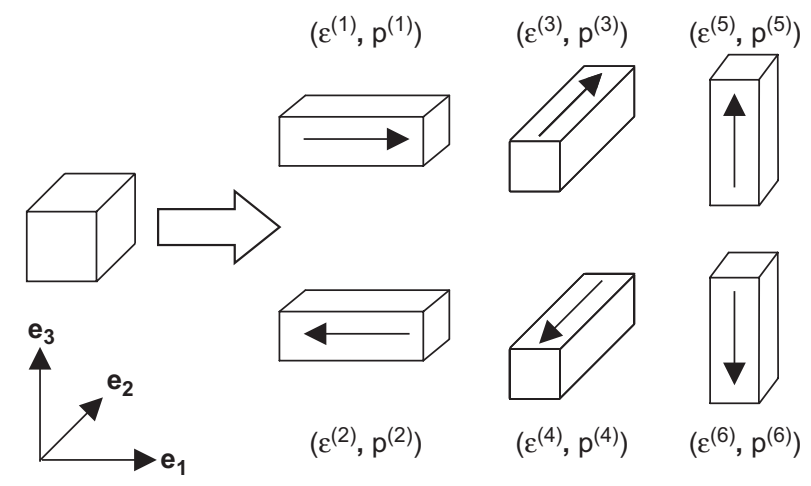

Fig. 1. Schematic of the six tetragonal ferroelectric variants $\left(\varepsilon^{(i)}, \mathbf{p}^{(i)}\right)$. The crystal and reference bases are assumed to coincide together, and each arrow represents the direction of polarization.

vector $\mathbf{x} \in \Omega$

$$
W(\boldsymbol{\varepsilon}(\mathbf{x}), \mathbf{p}(\mathbf{x})) \begin{cases}=0 & \text { if }(\boldsymbol{\varepsilon}(\mathbf{x}), \mathbf{p}(\mathbf{x})) \in \mathscr{Z}=\bigcup_{i=1}^{N}\left\{\left(\boldsymbol{\varepsilon}^{(i)}, \mathbf{p}^{(i)}\right)\right\} . \\ >0 & \text { otherwise. }\end{cases}
$$

For ferroelectric single crystals in the tetragonal phase, $N=6$, and the six variants can be expressed by

$$
\begin{aligned}
\boldsymbol{\varepsilon}^{(1)}=\left(\eta_{1}-\eta_{2}\right) \mathbf{e}_{1} \otimes \mathbf{e}_{1}+\eta_{2} \mathbf{I}, & \mathbf{p}^{(1)}=P_{\mathrm{s}} \mathbf{e}_{1}, \\
\boldsymbol{\varepsilon}^{(2)}=\left(\eta_{1}-\eta_{2}\right) \mathbf{e}_{1} \otimes \mathbf{e}_{1}+\eta_{2} \mathbf{I}, & \mathbf{p}^{(2)}=-P_{\mathrm{s}} \mathbf{e}_{1}, \\
\boldsymbol{\varepsilon}^{(3)}=\left(\eta_{1}-\eta_{2}\right) \mathbf{e}_{2} \otimes \mathbf{e}_{2}+\eta_{2} \mathbf{I}, & \mathbf{p}^{(3)}=P_{\mathrm{s}} \mathbf{e}_{2}, \\
\boldsymbol{\varepsilon}^{(4)}=\left(\eta_{1}-\eta_{2}\right) \mathbf{e}_{2} \otimes \mathbf{e}_{2}+\eta_{2} \mathbf{I}, & \mathbf{p}^{(4)}=-P_{\mathrm{s}} \mathbf{e}_{2}, \\
\boldsymbol{\varepsilon}^{(5)}=\left(\eta_{1}-\eta_{2}\right) \mathbf{e}_{3} \otimes \mathbf{e}_{3}+\eta_{2} \mathbf{I}, & \mathbf{p}^{(5)}=P_{\mathrm{s}} \mathbf{e}_{3}, \\
\boldsymbol{\varepsilon}^{(6)}=\left(\eta_{1}-\eta_{2}\right) \mathbf{e}_{3} \otimes \mathbf{e}_{3}+\eta_{2} \mathbf{I}, & \mathbf{p}^{(6)}=-P_{\mathrm{s}} \mathbf{e}_{3},
\end{aligned}
$$

where $\eta_{1}$ and $\eta_{2}$ are measured parameters for spontaneous strain, $P_{\mathrm{s}}$ for spontaneous polarization, and $\mathbf{e}_{1}=(1,0,0), \mathbf{e}_{2}=(0,1,0), \mathbf{e}_{3}=(0,0,1)$. Above $\mathbf{a} \otimes \mathbf{b}$ is the tensor product of two vectors $\mathbf{a}$ and $\mathbf{b}$. The schematic view of these six tetragonal variants $\left(\varepsilon^{(i)}, \mathbf{p}^{(i)}\right)$ are depicted in Fig. 1. Other cases such as rhombohedral $(N=8)$ and orthorhombic $(N=12)$ ferroelectric variants can be found in the work by Shu and Bhattacharya (2001) for details.

\subsection{Multirank laminated domain patterns}

The multiwell structure of the stored energy density $W$ in Eq. (3) gives rise to minimizers or domains on which strain and polarization are approximately constant and close to one of the ground states. To see it, consider a ferroelectric single crystal in the absence of external field and loading; i.e., $\mathbf{E}^{*}=\mathbf{0}$ and $\boldsymbol{\sigma}^{*}=\mathbf{0}$ in Eq. (1). The remaining terms in Eq. (1) are non-negative, and therefore, energy is minimized if each term is minimized individually. According to Eq. (3), the stored energy density $W$ is minimized if at each point $\mathbf{x} \in \Omega$ $(\boldsymbol{\varepsilon}(\mathbf{x}), \mathbf{p}(\mathbf{x})) \in \mathscr{Z}$. Next, minimizing the depolarization energy requires that the depolarization field is zero, and this in turn requires $\mathbf{p}$ to be divergence-free: $\nabla \cdot \mathbf{p}(\mathbf{x})=0$ on $\mathbb{R}^{3}$. The most obvious solutions satisfying these two requirements simultaneously are piecewise constant solutions; i.e., mixtures of ferroelectric variants separated by walls. However, these walls cannot be arbitrary since the jumps of strain and polarization across the interface are restricted to those that can maintain the interface unbroken and uncharged. To be precise, let $\mathbf{n}$ be the unit vector normal to an interface separating regions occupied by variants $\left(\varepsilon^{(i)}, \mathbf{p}^{(i)}\right)$ and $\left(\varepsilon^{(j)}, \mathbf{p}^{(j)}\right)$, respectively. The mechanical and electrical compatibility conditions across the interface are given by 
(Shu and Bhattacharya, 2001)

$$
\begin{aligned}
& \boldsymbol{\varepsilon}^{(i)}-\boldsymbol{\varepsilon}^{(j)}=\frac{1}{2}(\mathbf{a} \otimes \mathbf{n}+\mathbf{n} \otimes \mathbf{a}), \\
& \left(\mathbf{p}^{(i)}-\mathbf{p}^{(j)}\right) \cdot \mathbf{n}=0
\end{aligned}
$$

for some vector a. These two compatibility conditions ensure that the underlying distortion and the normal component of polarization vector are continuous across the interface. Violating these conditions will give rise to additional elastic and depolarization energies.

If both Eqs. (5) and (6) are satisfied, a laminated domain pattern can be formed with overall strain and polarization given by

$$
\langle\boldsymbol{\varepsilon}\rangle=\mu \boldsymbol{\varepsilon}^{(i)}+(1-\mu) \boldsymbol{\varepsilon}^{(j)}, \quad\langle\mathbf{p}\rangle=\mu \mathbf{p}^{(i)}+(1-\mu) \mathbf{p}^{(j)},
$$

where $\langle\cdots\rangle$ denotes the volume average and $0 \leqslant \mu \leqslant 1$ is the volume fraction of the $i$ th variant. If more than two variants coexist in a crystal, domains form highly characteristic and complicated patterns. While there are various candidates for domain patterns, an important one is a multirank laminate observed often in experiments. For example, rank-one lamellar and rank-two banded domains are commonly observed patterns in $\mathrm{BaTiO}_{3}$ (Arlt and Sasko, 1980; Wada et al., 1999). Further, it has been shown that, given any arbitrary average states of strain and polarization, a minimum energy configuration of domains which accommodate these prescribed averages can be constructed through multirank lamination (Li and Liu, 2004). Therefore, compatible multirank laminated domain patterns provide a low-energy path for domain switching, and the understanding of them leads to novel strategies of large strain actuation. In the following, an example of multirank laminates is investigated and will be used for illustrating the switching process.

Consider a ferroelectric crystal in the tetragonal phase with four coexisting variants (1), (2), (5) and (6), according to the labeling of Eq. (4). Fig. 2(a) shows a rank-3 laminate consisting of alternating sublayers L and $\mathrm{R}$ separated by an interface with unit normal $\mathbf{n}^{(\mathrm{III})}$, and $\mu_{3}$ is the volume fraction of sublayer L. To understand the lamination process of this multirank laminate, first consider the sublayer $\mathrm{L}$ which is a rank-2 laminate comprising variants (1), (2) and (5). Among them, variants (1) and (2) form a $180^{\circ}$ rank-1 laminate, while variants (1) and (5) form a $90^{\circ}$ domain pattern. These two rank-1 laminates can further form a compatible rank-2 domain pattern if the compatibility conditions given by Eqs. (5) and (6) are interpreted in the sense of average (Shu and Bhattacharya, 2001). In the present case, the average compatibility requires $\mathbf{n}^{(\mathrm{II})}=\left(\frac{1}{\sqrt{2}}, 0,-\frac{1}{\sqrt{2}}\right)$. Similarly, the sublayer $\mathrm{R}$ is another compatible rank-2 laminate consisting of variants (1), (2) and (6). Both sublayers R and L are characterized by local volume fractions $\mu_{1}$ and $\mu_{2}$. Now Fig. 2(a) is a legitimate domain pattern if sublayers $\mathrm{R}$ and L satisfy the compatibility conditions

a

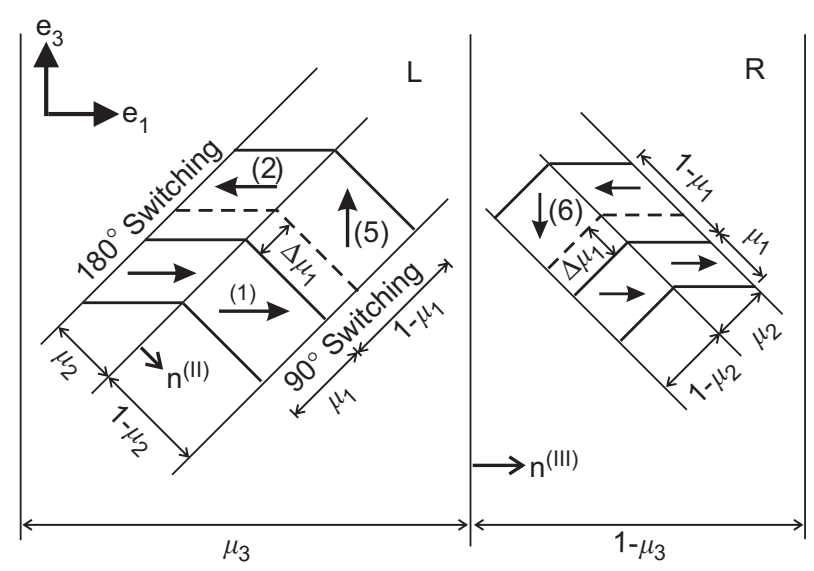

b

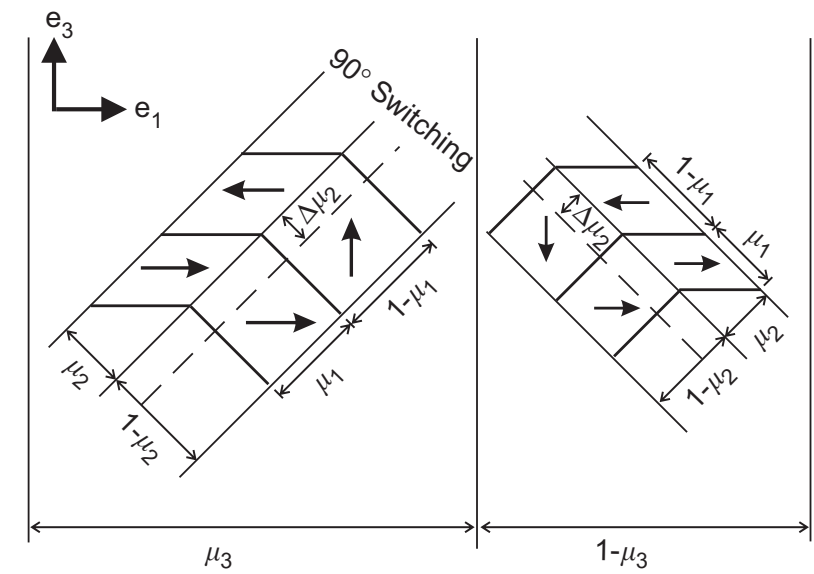

Fig. 2. A compatible rank-3 laminated domain pattern consists of tetragonal variants (1), (2), (5) and (6). Illustrated in (a) shows domain wall movements in the first rank laminate, while in (b) shows wall movements at the level of the second rank laminate. Notice that the change in $\mu_{1}$ induces two types of domain wall movements, while the change in $\mu_{2}$ induces only $90^{\circ}$ domain wall movements. 
across the interface with normal $\mathbf{n}^{(\mathrm{III})}$ in the sense of average. This is obvious by the present construction, since variants (5) and (6) can form a lamellar $180^{\circ}$ domain with an interfacial unit normal $\mathbf{n}^{(\mathrm{III})}=(1,0,0)$. Finally, the overall strain and polarization of this rank-3 laminated domain pattern can be obtained using Eq. (7) repeatedly in each level of laminates

$$
\begin{aligned}
& \langle\boldsymbol{\varepsilon}\rangle=\mu_{1} \boldsymbol{\varepsilon}^{(1)}+\mu_{2}\left(1-\mu_{1}\right) \boldsymbol{\varepsilon}^{(2)}+\mu_{3}\left(1-\mu_{1}\right)\left(1-\mu_{2}\right) \boldsymbol{\varepsilon}^{(5)}+\left(1-\mu_{1}\right)\left(1-\mu_{2}\right)\left(1-\mu_{3}\right) \boldsymbol{\varepsilon}^{(6)}, \\
& \langle\mathbf{p}\rangle=\mu_{1} \mathbf{p}^{(1)}+\mu_{2}\left(1-\mu_{1}\right) \mathbf{p}^{(2)}+\mu_{3}\left(1-\mu_{1}\right)\left(1-\mu_{2}\right) \mathbf{p}^{(5)}+\left(1-\mu_{1}\right)\left(1-\mu_{2}\right)\left(1-\mu_{3}\right) \mathbf{p}^{(6)} .
\end{aligned}
$$

\subsection{Domain switching}

Consider a bulk ferroelectric crystal subjected to combined uniform electromechanical loads; i.e. both $\mathbf{E}^{*}$ and $\boldsymbol{\sigma}^{*}$ are constant in Eq. (1). If the well structure of $W(\boldsymbol{\varepsilon}, \mathbf{p})$ in Eq. (3) is steep away from the ground states, the state of strain and polarization may assume to be restricted on the well points for most of points in the body $\Omega$. This leads to the constrained theory of ferroelectrics, originating from the study of shape-memory alloys (James, 1986) and magnetostrictive materials (DeSimone and James, 2002), respectively. Currently, this concept has also been extended to the study of ferromagnetic shape-memory alloys ( $\mathrm{Ma}$ and $\mathrm{Li}, 2007)$. Moreover, if the domain patterns are compatible throughout the crystal, the source of depolarization field comes only from the unshielded boundary of the body, and not from the interior. Combining all these features, the total potential energy per unit volume in Eq. (1) can be simplified to be

$$
\langle I\rangle=-\mathbf{E}^{*} \cdot\langle\mathbf{p}\rangle-\boldsymbol{\sigma}^{*} \cdot\langle\boldsymbol{\varepsilon}\rangle+\frac{1}{2 \varepsilon_{0}} \mathbf{K}\langle\mathbf{p}\rangle \cdot\langle\mathbf{p}\rangle,
$$

where $\mathbf{K}$ is some semipositive-definite symmetric tensor which is related to the geometry of the crystal and the arrangement of the electrodes (Bhattacharya et al., 2008). Finally, we assume that the compatible domain patterns are those obtained by multirank lamination described in Section 2.2. As a result, the potential energy density in Eq. (9) can be expressed explicitly in terms of local volume fractions $\boldsymbol{\mu}=\left(\mu_{1}, \mu_{2}, \ldots, \mu_{N-1}\right)$; i.e., $\langle I\rangle=\langle I\rangle(\boldsymbol{\mu})$.

Now we turn to the kinetics of domain switching. The reduction in potential energy provides the thermodynamic driving force for domain evolution. Fig. 2 demonstrates that the domain evolution results in the change of local volume fraction $\mu_{j}$ in the $j$ th-rank laminate. Thus, the driving force needed to move the major interface in the $j$ th-rank laminate is defined by

$$
F_{j}(\boldsymbol{\mu})=-\frac{\partial}{\partial \mu_{j}}\langle I\rangle(\boldsymbol{\mu}) .
$$

Nevertheless, domain switching is resisted by the dissipative motion of domain walls (Huber et al., 1999). As a result, domain switching occurs when

$$
-F_{j}(\boldsymbol{\mu}) \mathrm{d} \mu_{j}+G_{j}^{\mathrm{c}}(\boldsymbol{\mu})\left|\mathrm{d} \mu_{j}\right| \leqslant 0,
$$

where $G_{j}^{\mathrm{c}}(\boldsymbol{\mu})$ is the non-negative critical resistance force in the $j$ th-rank laminate and the summation over the index $j$ implied in Eq. (11) can be removed due to the independence of $\mu_{j}$. The explicit expression of $G_{j}^{\mathrm{c}}(\boldsymbol{\mu})$ can be obtained in terms of specific types of domain wall movements, and we illustrate it using the switching system $\left\{\left(\boldsymbol{\varepsilon}^{(i)}, \mathbf{p}^{(i)}\right), i=1,2,5,6\right\}$ as an example. The general result is provided in Section 2.5. Consider the domain wall movements in Fig. 2. First assuming local volume fractions $\mu_{2}$ and $\mu_{3}$ are fixed. As demonstrated in Fig. 2(a), a change in $\mu_{1}$ will involve $90^{\circ}$ switching in the sublayer with local volume fraction $\left(1-\mu_{2}\right)$ and $180^{\circ}$ switching in another sublayer with local volume fraction $\mu_{2}$. Thus

$$
G_{1}^{\mathrm{c}}=\gamma_{1}^{(1)} \mu_{2}+\gamma_{2}^{(1)}\left(1-\mu_{2}\right),
$$

where $\gamma_{1}^{(1)}$ and $\gamma_{2}^{(1)}$ are coefficients associated with energy dissipation due to the movements of $180^{\circ}$ and $90^{\circ}$ walls in the first rank laminate. Next, as $\mu_{2}$ changes while $\mu_{1}$ and $\mu_{3}$ are kept fixed, Fig. 2(b) demonstrates that a $90^{\circ}$ switching occurs in the local volume fraction $\left(1-\mu_{1}\right)$. This gives

$$
G_{2}^{\mathrm{c}}=\gamma_{2}^{(2)}\left(1-\mu_{1}\right)
$$


where $\gamma_{2}^{(2)}$ is the dissipation coefficient associated with the movements of $90^{\circ}$ walls in the second rank laminate. Finally, consider the change in $\mu_{3}$ while others are kept constant. The net change in polarization between the layers $\mathrm{L}$ and $\mathrm{R}$ is $\left(1-\mu_{1}\right)\left(1-\mu_{2}\right)\left(\mathbf{p}^{(5)}-\mathbf{p}^{(6)}\right)$, which corresponds to $180^{\circ}$ domain switching when the interface separating the sublayers $\mathrm{L}$ and $\mathrm{R}$ moves. Therefore

$$
G_{3}^{\mathrm{c}}=\gamma_{1}^{(3)}\left(1-\mu_{1}\right)\left(1-\mu_{2}\right),
$$

where $\gamma_{1}^{(3)}$ is the dissipation coefficient related to moving $180^{\circ}$ walls in the third rank laminate.

The term $G_{j}^{\mathrm{c}}$ can be also viewed as the work per unit volume dissipated in moving the interfaces in the $j$ thrank of laminate. It can be determined accounting for the net change in polarization between alternating layers in each $j$ th-rank laminate. The total work cost per unit volume in domain switching is then given by the corresponding dissipation coefficients multiplied by the local volume fractions involved in the process. In addition, if the dissipation coefficients are not distinguishable among different levels of laminates, $\gamma_{1}^{(j)}=\gamma_{1}$ and $\gamma_{2}^{(j)}=\gamma_{2}$, and can be determined experimentally in terms of the coercive fields $E_{180^{\circ}}^{\mathrm{c}}$ and $E_{90^{\circ}}^{\mathrm{c}}$ which are required for $180^{\circ}$ and $90^{\circ}$ domain switchings. To see that, assume that the polarization and strain are uniform in the crystal. Suppose the crystal is subjected to only a uniaxial electric field $\mathbf{E}^{*}$ applied in the opposite direction of polarization. As the magnitude of the field reaches a critical value $E_{180^{\circ}}^{\mathrm{c}}$, a $180^{\circ}$ switching in polarization occurs, and the work per unit volume done by this external electric field is therefore

$$
\mathbf{E}^{*} \cdot \Delta \mathbf{p}_{180^{\circ}}=2 P_{\mathrm{s}} E_{180^{\circ}}^{\mathrm{c}} \equiv \gamma_{1} .
$$

Similarly, let the external electric field be applied perpendicular to the direction of polarization. When the field strength is increased to a critical value $E_{90^{\circ}}^{\mathrm{c}}$, it will cause the polarization to switch $90^{\circ}$. Again, to accomplish this process, the work per unit volume done by this external electric field is

$$
\mathbf{E}^{*} \cdot \Delta \mathbf{p}_{90^{\circ}}=P_{\mathrm{s}} E_{90^{\circ}}^{\mathrm{c}} \equiv \gamma_{2} .
$$

\subsection{Flat-plate configuration}

Consider a ferroelectric single crystal in the shape of a flat plate with electrodes on the top and bottom surfaces as shown in Fig. 3. We call this setup the "flat-plate configuration" which is the most common design in ferroelectric devices. The crystal in this configuration is subjected to a constant uniaxial compressive stress and an external cyclic electric field. Both are applied in the direction perpendicular to the plate face and therefore, can be written as

$$
\boldsymbol{\sigma}^{*}=\sigma_{33}^{*} \mathbf{e}_{3} \otimes \mathbf{e}_{3}, \quad \mathbf{E}^{*}=E_{3}^{*} \mathbf{e}_{3},
$$

where $\sigma_{33}^{*}$ is a constant dead load and $E_{3}^{*}$ varies cyclically.

The crystal and reference bases are assumed to coincide together in this case; i.e., the pseudocubic [0 01 l] axis is normal to the plate face. The ferroelectric phase in the temperature range of interest is tetragonal under the present consideration. According to Eqs. (4) and (17), $-\boldsymbol{\sigma}^{*} \cdot\left\langle\boldsymbol{\varepsilon}^{(i)}\right\rangle=-\sigma_{33}^{*} \eta_{2}<0$ for $i=1, \ldots, 4$, while $-\boldsymbol{\sigma}^{*}$.

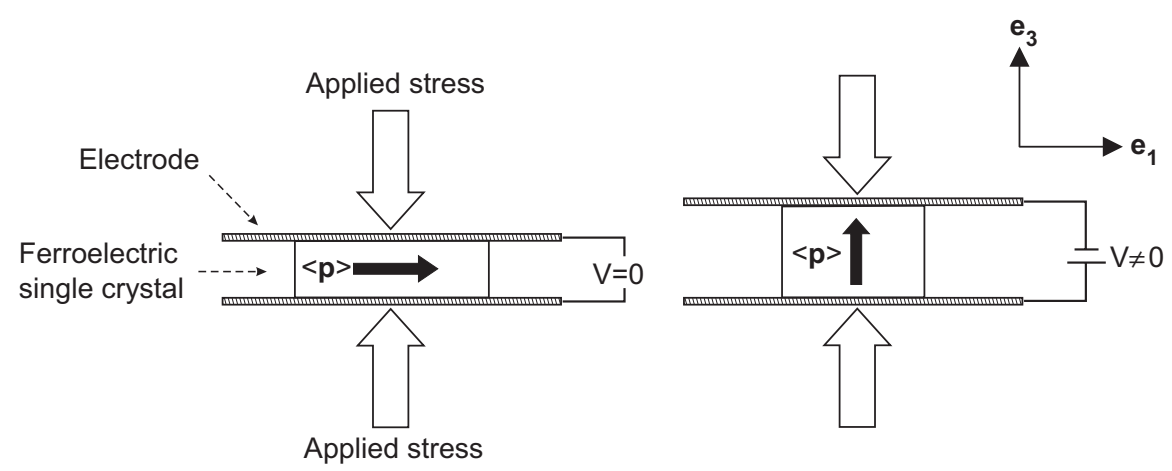

Fig. 3. Schematic of a flat-plate configuration. 
$\left\langle\boldsymbol{\varepsilon}^{(5)}\right\rangle=-\boldsymbol{\sigma}^{*} \cdot\left\langle\boldsymbol{\varepsilon}^{(6)}\right\rangle=-\sigma_{33}^{*} \eta_{1}>0$ since $\sigma_{33}^{*}<0, \eta_{1}>0$ and $\eta_{2}<0$. Thus, from the consideration of minimizing energy given by Eq. (9), the polarization prefers to be in the in-plane state at sufficiently large compressive stress and small electric field. As the magnitude of the electric field increases, the crystal prefers the [ $\left.\begin{array}{lll}0 & 0 & 1\end{array}\right]$ polarized state since $-\mathbf{E}^{*} \cdot \mathbf{p}^{(5)}=-E_{3}^{*} P_{\mathrm{s}}<0$ and $-\mathbf{E}^{*} \cdot \mathbf{p}^{(i)} \geqslant 0$ otherwise if $E_{3}^{*}>0$. However, this switching is resisted by the compressive stress, and there is an exchange of stability at the critical field above which the switching occurs. Finally, the polarization switches back to the in-plane state when the field strength decreases, giving rise to a significant out-of-plane shape change of the specimen, as demonstrated in Fig. 3. Ideally this stress-activated domain switching provides an actuation strain as high as $\left(\eta_{1}-\eta_{2}\right)$ for this soft device (Shu and Bhattacharya, 2001), and we now study the details here.

The dimensions of a ferroelectric crystal considered here are $L \times L \times H$, where $L$ is the width of the edge and $H$ is the thickness. As described above, when the load is kept fixed and field is cycled, the [0 01$]$ polarized state can be switched to either [ 100$]$ or $\left[\begin{array}{lll}0 & 1 & 0\end{array}\right]$ polarized states with equal probability. This can be achieved through either the switching system $\left\{\left(\boldsymbol{\varepsilon}^{(i)}, \mathbf{p}^{(i)}\right), i=1,2,5,6\right\}$ or another one comprising $\left\{\left(\varepsilon^{(i)}, \mathbf{p}^{(i)}\right), i=3,4,5,6\right\}$. As both have the same potential energy in Eq. (9) for this flat-plate configuration, the problem can be simplified if only the switching system $\left\{\left(\boldsymbol{\varepsilon}^{(i)}, \mathbf{p}^{(i)}\right), i=1,2,5,6\right\}$ is involved for investigating the electrostrictive response of this device. For other complicated configurations, all variants are needed to be included, and we study it in Section 2.5 .

Under this assumption, the potential energy density in Eq. (9) is reduced to

$$
\langle I\rangle=-E_{3}^{*}\left\langle p_{3}\right\rangle-\sigma_{33}^{*}\left\langle\varepsilon_{33}\right\rangle+\frac{K}{2 \varepsilon_{0}}\left\langle p_{1}\right\rangle^{2}
$$

for this soft device. Above, $K$ is the depolarization constant for a rectangular geometry with thickness $H$ and width $L$, and is given by

$$
K \approx 8 \frac{H}{L} \sum_{n=1}^{\infty} \frac{\left[1-(-1)^{n}\right]}{n^{3} \pi^{3}}\left(1-\mathrm{e}^{-n \pi L / H}\right),
$$

where the derivation of Eq. (19) is provided in Appendix A. In the last term of Eq. (18), the contribution from $\left\langle p_{3}\right\rangle$ is not included due to the electrical shielding on the top and bottom faces of the crystal. As discussed in Section 2.2, these four variants $\left\{\left(\boldsymbol{\varepsilon}^{(i)}, \mathbf{p}^{(i)}\right), i=1,2,5,6\right\}$ considered here can form a compatible rank-3 laminate, as shown in Fig. 2. The strain and polarization averages of this laminate are given by Eq. (8). Thus, the only non-zero components of polarization average in terms of local volume fractions $\mu_{j}$ are given by

$$
\begin{aligned}
& \left\langle p_{1}\right\rangle=\left[\mu_{1}-\left(1-\mu_{1}\right) \mu_{2}\right] P_{\mathrm{s}}, \\
& \left\langle p_{3}\right\rangle=\left(1-\mu_{1}\right)\left(1-\mu_{2}\right)\left(2 \mu_{3}-1\right) P_{\mathrm{s}},
\end{aligned}
$$

and the 33-component of the strain average is given by

$$
\left\langle\varepsilon_{33}\right\rangle=\left(1-\mu_{1}\right)\left(1-\mu_{2}\right) \eta_{1}+\left[\mu_{1}+\left(1-\mu_{1}\right) \mu_{2}\right] \eta_{2} .
$$

The switching criterion for this switching system is given by Eq. (11) discussed in Section 2.3. But for this flatplate configuration, it can be further simplified to be

$$
-\tilde{F}_{j}(\boldsymbol{\mu}) \mathrm{d} \mu_{j}+\tilde{G}_{j}^{\mathrm{c}}(\boldsymbol{\mu})\left|\mathrm{d} \mu_{j}\right| \leqslant 0
$$

where from Eq. (10),

$$
\begin{aligned}
\tilde{F}_{1}= & -E_{3}^{*} P_{\mathrm{s}}\left(1-\mu_{2}\right)\left(2 \mu_{3}-1\right)+\sigma_{33}^{*}\left(\eta_{2}-\eta_{1}\right)\left(1-\mu_{2}\right) \\
& -\frac{K}{\varepsilon_{0}} P_{\mathrm{s}}^{2}\left[\mu_{1}-\left(1-\mu_{1}\right) \mu_{2}\right]\left(1+\mu_{2}\right), \\
\tilde{F}_{2}= & -E_{3}^{*} P_{\mathrm{s}}\left(2 \mu_{3}-1\right)+\sigma_{33}^{*}\left(\eta_{2}-\eta_{1}\right)+\frac{K}{\varepsilon_{0}} P_{\mathrm{s}}^{2}\left[\mu_{1}-\left(1-\mu_{1}\right) \mu_{2}\right], \\
\tilde{F}_{3}= & 2 E_{3}^{*} P_{\mathrm{s}}
\end{aligned}
$$


and from Eqs. (12)-(14),

$$
\begin{aligned}
& \tilde{G}_{1}^{\mathrm{c}}=\gamma_{1}^{(1)} \mu_{2}+\gamma_{2}^{(1)}\left(1-\mu_{2}\right), \\
& \tilde{G}_{2}^{\mathrm{c}}=\gamma_{2}^{(2)}, \\
& \tilde{G}_{3}^{\mathrm{c}}=\gamma_{1}^{(3)},
\end{aligned}
$$

since the common term $\left(1-\mu_{1}\right)$ is eliminated on both sides of $F_{2}$ and $G_{2}^{\mathrm{c}}$, and another common term $\left(1-\mu_{1}\right)\left(1-\mu_{2}\right)$ is also eliminated on both sides of $F_{3}$ and $G_{3}^{\mathrm{c}}$. Finally, the local volume fractions $\mu_{1}, \mu_{2}$ and $\mu_{3}$ can be determined via Eqs. (22)-(24) under cyclic $E_{3}^{*}$ at fixed $\sigma_{33}^{*}$, and we will demonstrate it in Section 4.

\subsection{Other configurations}

For general configurations without any symmetry in geometry and loadings, the switching system should involve all variants in order to capture the complete electromechanical response of a ferroelectric crystal under loadings. For tetragonal ferroelectrics, it has been shown by Li and Liu (2004) that the lowest rank of laminate satisfying any prescribed averages of spontaneous strain and polarization is a rank-5 laminated domain pattern. In this case, the strain and polarization averages can be expressed in terms of local volume fractions $\boldsymbol{\mu}$ by

$$
\begin{aligned}
& \langle\boldsymbol{\varepsilon}\rangle=\mu_{1} \boldsymbol{\varepsilon}^{(1)}+\mu_{2}\left(1-\mu_{1}\right) \boldsymbol{\varepsilon}^{(2)}+\cdots+\mu_{5} \prod_{k=1}^{4}\left(1-\mu_{k}\right) \boldsymbol{\varepsilon}^{(5)}+\prod_{k=1}^{5}\left(1-\mu_{k}\right) \boldsymbol{\varepsilon}^{(6)}, \\
& \langle\mathbf{p}\rangle=\mu_{1} \mathbf{p}^{(1)}+\mu_{2}\left(1-\mu_{1}\right) \mathbf{p}^{(2)}+\cdots+\mu_{5} \prod_{k=1}^{4}\left(1-\mu_{k}\right) \mathbf{p}^{(5)}+\prod_{k=1}^{5}\left(1-\mu_{k}\right) \mathbf{p}^{(6)} .
\end{aligned}
$$

Substituting Eqs. (25) and (26) into Eqs. (9) and (10) gives the thermodynamic driving force in terms of local volume fraction $\boldsymbol{\mu}$.

The switching criterion is the same as that in Eq. (11) except that the critical dissipation functions are given by (Yen, 2008)

$$
\begin{aligned}
& G_{1}^{\mathrm{c}}=\gamma_{1}^{(1)} \mu_{2}+\gamma_{2}^{(1)}\left(1-\mu_{2}\right), \\
& G_{2}^{\mathrm{c}}=\gamma_{2}^{(2)}\left(1-\mu_{1}\right), \\
& G_{3}^{\mathrm{c}}=\left[\gamma_{1}^{(3)} \mu_{4}+\gamma_{2}^{(3)}\left(1-\mu_{4}\right)\right]\left(1-\mu_{1}\right)\left(1-\mu_{2}\right), \\
& G_{4}^{\mathrm{c}}=\gamma_{2}^{(4)}\left(1-\mu_{1}\right)\left(1-\mu_{2}\right)\left(1-\mu_{3}\right), \\
& G_{5}^{\mathrm{c}}=\gamma_{1}^{(5)}\left(1-\mu_{1}\right)\left(1-\mu_{2}\right)\left(1-\mu_{3}\right)\left(1-\mu_{4}\right),
\end{aligned}
$$

where $\gamma_{1}^{(j)}$ and $\gamma_{2}^{(j)}$ are dissipation coefficients associated with moving $180^{\circ}$ and $90^{\circ}$ walls in the $j$ th-rank laminate. Finally, they can be simplified if they cannot be distinguished in any levels of the laminate. This gives $\gamma_{1}^{(j)}=\gamma_{1}=2 E_{180^{\circ}}^{\mathrm{c}} P_{\mathrm{s}}$ and $\gamma_{2}^{(j)}=\gamma_{2}=E_{90^{\circ}}^{\mathrm{c}} P_{\mathrm{s}}$ as discussed in Section 2.3.

\subsection{Model comparisons}

Many approaches have been proposed to model the nonlinear electromechanical behavior of ferroelectric materials. One of the most successful models is the one developed by Huber et al. (1999). The framework proposed here is similar to theirs from the point of view of describing the switching processes at the crystal lattice scale. But the two differ in the way of specifying the kinematic descriptions of switching variants and the criterions for domain switching. We now briefly describe the differences here. 
First, the internal or kinematic variables that Huber et al. choose are incremental volume fractions of variants switched from domain $i$ to another domain $j$, and are denoted by $\dot{f}_{i j}$. The changes in remanent strain and polarization due to $\dot{f}_{i j}$ are not arbitrary due to Eqs. (5) and (6). According to the present model, the satisfaction of these two equations ensures that the wall separating the domains $i$ and $j$ is compatible, while Huber et al.'s model interprets it in terms of the language of crystal plasticity. For example, for the remanent strain, the vector a in Eq. (5) is normalized to be the direction of the simple shear on the plane of unit normal $\mathbf{n}$ and vice versa. As a result, this indicates that the switching process from domain $i$ to domain $j$ follows a locally compatible path in their model. However, the overall compatibility may not be satisfied in general if all the switching systems are involved. In contrast, the present framework chooses a set of local volume fractions $\boldsymbol{\mu}=\left(\mu_{1}, \mu_{2}, \ldots, \mu_{N-1}\right)$ to assure that the underlying multirank laminates remain compatible during evolution. In addition, the incremental changes of strain and polarization averages can be obtained through Eqs. (25) and (26) in terms of $\boldsymbol{\mu}$ and $\dot{\boldsymbol{\mu}}$.

Next, the switching criterions in both models require balancing the thermodynamical driving and resistance forces. The driving forces typically contain terms owing to electromechanical loadings. But Huber et al.'s model includes additional terms due to changes occurring in the elastic, piezoelectric or dielectric moduli on switching, while the present model neglects them because of the constrained assumptions on the ground states. However, the effect of depolarization energy is only considered in the present model.

Finally, in Huber et al.'s model, the critical resistance force $G_{i j}^{\mathrm{c}}$ for each transformation system represents the work per unit volume dissipated in moving domain wall separating variant $i$ and variant $j$. While in the present model, the critical resistance force $G_{j}^{\mathrm{c}}$ is work per unit volume dissipated in moving the interfaces in the $j$ th-rank of laminate. It can be determined by the consideration of net change in polarization between alternating layers. In addition, the present lamination construction provides a simple way of specifying the different types of domain wall movements, leading to the explicit expression of $G_{j}^{\mathrm{c}}$ as in Eq. (24) or (27).

\section{Experiment under flat-plate configuration}

An experiment to study the electromechanical behavior of $\mathrm{BaTiO}_{3}$ single crystals has been performed under the flat-plate configuration as described in Section 2.4. A number of (001)-oriented cuboidal $\mathrm{BaTiO}_{3}$ single crystals measuring $5 \mathrm{~mm} \times 5 \mathrm{~mm} \times 2 \mathrm{~mm}$ have been obtained from Superconix Inc., MN, USA. Electrodes are produced on the $5 \mathrm{~mm} \times 5 \mathrm{~mm}$ surfaces. A loading fixture has been developed for the simultaneous application of compressive stress and electric field (see Fig. 4 in Shieh et al., 2003). It consists of brass plates which provide electrical contact to the electrode surfaces of the crystal, and nylon spacers to insulate the crystal from the universal test frame which supplies the mechanical loading. The upper brass plate is connected to ground via a ferroelectric analyzer for charge measurement. The lower brass plate is connected to a high-voltage amplifier which supplies the electrical loading. Miniature strain gauges are attached onto the $5 \mathrm{~mm} \times 2 \mathrm{~mm}$ faces of the crystal to measure strain changes in the loading direction. The entire loading fixture is supported in a silicon oil bath to prevent breakdown arcing. The details of the experimental setup and measuring apparatuses for the hysteresis measurements can be found in Shieh and Shu (2008).

The experimental butterfly curves (strain vs. electric field) for the present $\mathrm{BaTiO}_{3}$ single crystal are shown in Fig. 4(a) measured at various compressive stresses ranging from 2.86 to $5.84 \mathrm{MPa}$. The applied electric field has an amplitude of $1.25 \mathrm{MVm}^{-1}$ and a frequency of $0.2 \mathrm{~Hz}$. Note that the strain measurement in the figure is the "actuation strain", that is, the minimum strain is used as the reference point and is calibrated to be zero following common practice. Besides the strain measurements, the two input parameters required for the theoretical framework, $E_{180^{\circ}}^{\mathrm{c}}$ and $E_{90^{\circ}}^{\mathrm{c}}$ of the single crystal, have also been obtained independently from Fig. 4(a). The coercive field $E_{180^{\circ}}^{\mathrm{c}}=0.23 \mathrm{MVm}^{-1}$ is measured from the polarization hysteresis at zero compressive stress, and $E_{90^{\circ}}^{\mathrm{c}}=0.26 \mathrm{MVm}^{-1}$ is obtained from the in situ domain observation using crossed polarizers under an arrangement where the applied electric field is perpendicular to the poling direction of the polished crystal. Finally, the relation between the coercive field $E_{90^{\circ}}^{\mathrm{c}}$ and coercive stress $\sigma^{\mathrm{c}}$ can be established via the energy argument as in Eq. (9)

$$
E_{90^{\circ}}^{\mathrm{c}} P_{\mathrm{s}}=\sigma^{\mathrm{c}}\left(\eta_{1}-\eta_{2}\right) \text {. }
$$


a

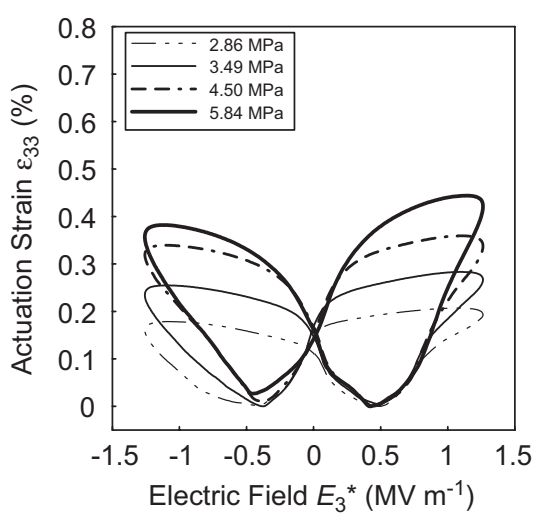

b

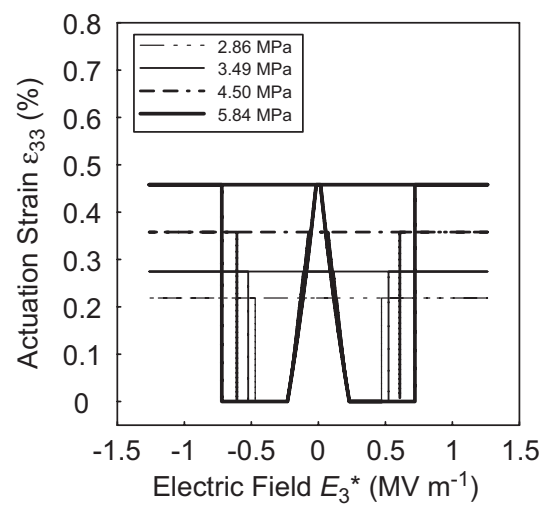

C

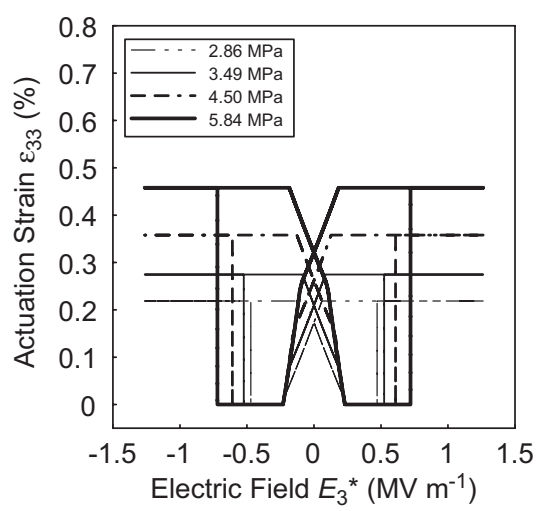

Fig. 4. Strain vs. electric field hysteresis curves for the $\mathrm{BaTiO}_{3}$ crystal under various compressive stresses. Notice that (a) is obtained from experiment, while (b) and (c), respectively, are obtained from simulation without and with the consideration of the $90^{\circ}$ onset and finish field points.

Table 1

Numerical input data used in Eqs. (22)-(24) under various cases I, II, III and IV

\begin{tabular}{|c|c|c|c|c|c|c|c|}
\hline & $E_{180^{\circ}}^{\mathrm{c}}$ & $E_{90^{\circ}}^{\mathrm{c}}$ & $\frac{\gamma_{1}^{(1)}}{2 E_{180^{\circ}}^{\mathrm{c}} P_{\mathrm{s}}}$ & $\frac{\gamma_{2}^{(1)}}{E_{90^{\circ}}^{\mathrm{c}} P_{\mathrm{s}}}$ & $\frac{\gamma_{2}^{(2)}}{E_{90^{\circ}}^{\mathrm{c}} P_{\mathrm{s}}}$ & $\frac{\gamma_{1}^{(3)}}{2 E_{180^{\circ}}^{\mathrm{c}} P_{\mathrm{s}}}$ & $K$ \\
\hline I & 0.23 & 0.26 & 1 & 1 & 1 & 1 & 0 \\
\hline II & 0.23 & 0.26 & 1 & 1 & 1 & 1 & Eq. (19) \\
\hline III & 0.23 & 0.26 & 3.5 & 1 & 1 & 1 & 0 \\
\hline IV & 0.23 & 0.26 & 3.5 & 1 & 1 & 1 & Eq. (19) \\
\hline
\end{tabular}

Note that the unit for coercive fields is $\mathrm{MVm}^{-1}$.

Using this relation gives the coercive stress $\sigma^{\mathrm{c}}=6.2 \mathrm{MPa}$ in the present crystal, since for $\mathrm{BaTiO}_{3}, \eta_{1}=0.67 \%$, $\eta_{2}=-0.42 \%$ and $P_{\mathrm{s}}=0.26 \mathrm{C} \mathrm{m}^{-2}$ (Jona and Shirane, 1962).

\section{Results and comparisons}

\subsection{Depolarization effect}

Fig. 4(a) shows that with the increase of compressive stress, the measured actuation strain increases significantly. It is mainly due to the enhancement of stress-activated $90^{\circ}$ domain switching. In addition, the high-elastic moduli of $\mathrm{BaTiO}_{3}$ (56-211 GPa, depending on the measured directions ( $\mathrm{Li}$ et al., 1991)) make certain that the measured strains, even at $5.84 \mathrm{MPa}$, are predominately transformation strains by domain switching, and the contribution of mechanical elastic strains can be ignored. However, it is also observed from the butterfly loops of Fig. 4(a) that the measured actuation strains are all far smaller than the theoretical maximum. Indeed, the theoretical maximum actuation strain, allowed by crystallography, is $\left(\eta_{1}-\eta_{2}\right)$ for tetragonal ferroelectric single crystals (Shu and Bhattacharya, 2001). Thus, for $\mathrm{BaTiO}_{3}$ crystals, this maximum is $1.09 \%$. The measured maximum actuation strain from Fig. 4(a), however, is only around $0.45 \%$ at $5.84 \mathrm{MPa}$. Certain visible microcracks have been observed within the crystal when the applied stress exceeds $5.84 \mathrm{MPa}$, leading to smaller strain.

The switching criterion proposed by Eq. (22) is employed to explain such a significant reduction in strain. The input parameters used for simulation include $E_{180^{\circ}}^{\mathrm{c}}=0.23 \mathrm{MVm}^{-1}$ and $E_{90^{\circ}}^{\mathrm{c}}=0.26 \mathrm{MVm}^{-1}$ measured from the present $\mathrm{BaTiO}_{3}$ crystal, as described in Section 3. The dissipation coefficients are assumed to be not distinguished in any levels of the laminate. With these parameters listed in Table 1 (cases I and II), the 
a

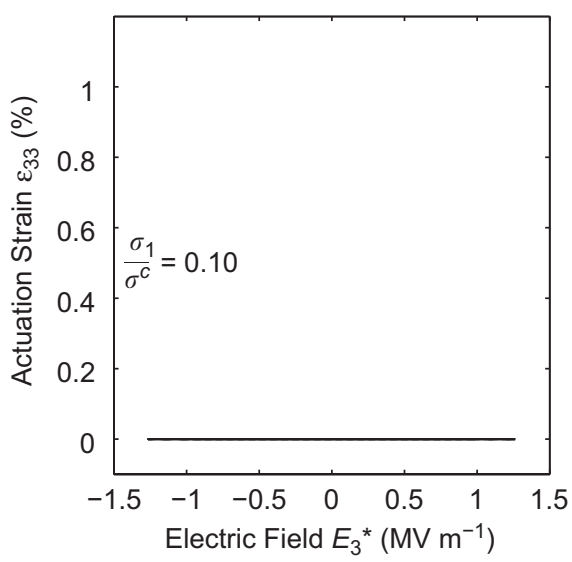

C

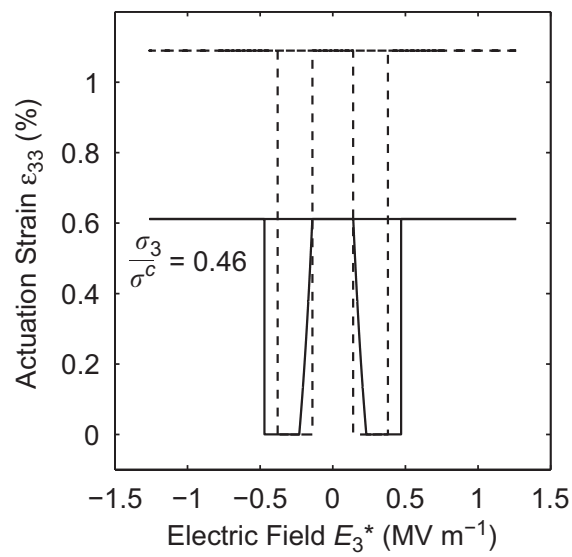

b

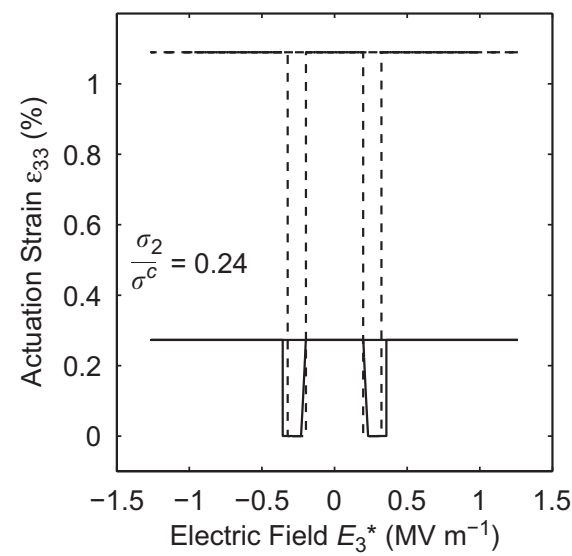

d

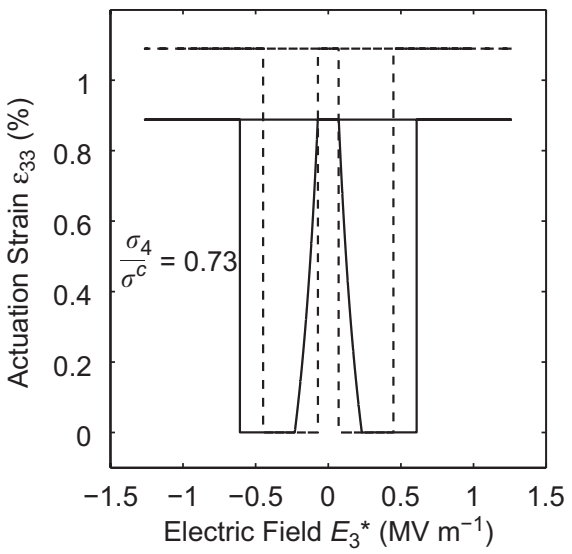

Fig. 5. Butterfly loops are simulated to demonstrate the effect of depolarization on actuation strains. The dashed/solid lines are the results excluding/including the depolarization effect, respectively. Note that $\sigma_{1}=0.6 \mathrm{MPa}, \sigma_{2}=1.5 \mathrm{MPa}, \sigma_{3}=2.86 \mathrm{MPa}, \sigma_{4}=4.5 \mathrm{MPa}$ and $\sigma^{\mathrm{c}}=6.2 \mathrm{MPa}$.

influence of different compressive stresses on the butterfly loops is demonstrated in Fig. 5 for various selected stress levels $\left(\sigma_{1}=0.6 \mathrm{MPa}, \sigma_{2}=1.5 \mathrm{MPa}, \sigma_{3}=2.86 \mathrm{MPa}\right.$ and $\left.\sigma_{4}=4.5 \mathrm{MPa}\right)$. In each of Figs. 5(a)-(d) there are two curves: the one with the dashed line neglects the depolarization effect $(K=0)$, while the other one with the solid line includes this influence $(K \neq 0)$. Apparently, except for very small compressive stresses, the actuation strain always reaches the theoretical maximum $1.09 \%$ if the depolarization effect is not taken into account. In contrast, if this effect is considered, an obvious strain reduction is observed in Fig. 5 as long as the compressive stress is smaller than the coercive stress $\sigma^{\mathrm{c}}$. The simulation results demonstrate that the depolarization field has a non-trivial effect which hinders the actuation strain from achieving its theoretical maximum.

To understand the wide difference as illustrated in Fig. 5, the driving and resistance forces for domain evolution described by Eqs. (23) and (24) are revisited analytically. The crystal initially prefers the [0 01 l] polarized state at large positive electric field. This corresponds to $\mu_{1}=\mu_{2}=0$ and $\mu_{3}=1$ according to Eq. (20). The switching starts when the field decreases and reaches the value determined by

$$
-P_{\mathrm{s}} E_{3}^{*}+\left(\eta_{2}-\eta_{1}\right) \sigma_{33}^{*}+\frac{K}{\varepsilon_{0}} P_{\mathrm{s}}\left\langle p_{1}\right\rangle=P_{\mathrm{s}} E_{90^{\circ}}^{\mathrm{c}} .
$$

Obviously if the depolarization effect is not taken into account; i.e., $K=0$ in Eq. (29), the $90^{\circ}$ switching process would be completed if the electromechanical loading is balanced by $P_{\mathrm{s}} E_{90^{\circ}}^{\mathrm{c}}$, and therefore, the 
actuation strain would achieve its maximum value $\left(\eta_{1}-\eta_{2}\right)$. Indeed, from Eq. (29) with $K=0$, the smallest compressive stress in the absence of depolarization effect such that above it the theoretical maximum strain can be achieved is

$$
\left|\sigma_{33}^{*}\right|=\left|\frac{P_{\mathrm{s}}\left(E_{90^{\circ}}^{\mathrm{c}}-E_{180^{\circ}}^{\mathrm{c}}\right)}{\eta_{2}-\eta_{1}}\right| .
$$

In the present case, it is $0.72 \mathrm{MPa}$ and the corresponding normalized stress ratio by coercive stress is 0.11 . Above this ratio, the actuation strains always attain to the maximum value $1.09 \%$ as can be seen from Fig. 5. On the other hand, if $K \neq 0$ in Eq. (29), the initiation of switching depends on $\left\langle p_{1}\right\rangle$, and the determination of it can be obtained by balancing the driving against resistance forces in Eqs. (23) and (24).

Next, a further examination of the simulated butterfly curves (solid lines) in Fig. 5 reveals that they only qualitatively agree with experimental measurements shown in Fig. 4(a). For example, the maximum actuation strains obtained from Figs. 5(c) to (d) are $0.61 \%$ and $0.88 \%$, respectively. They are larger than the measured strains as shown in Fig. 4(a) at the identical level of compressive stresses 2.86 and $4.50 \mathrm{MPa}$. It suggests that the depolarization-induced hardening is unlikely the sole reason for the pronounced reduction in actuation strain observed in experiment. Indeed, Fig. 5 is obtained assuming dissipation coefficients are not distinguishable among different levels of laminates. However, the construction of a multirank laminate assumes that the scales are widely separated, leading to much finer and many more twin structures in the lowest rank of laminate. Intuitively, it may imply that the wall movements in the lowest rank of laminate cost more energy dissipation than in other higher ranks of laminate. To account for that, Fig. 6 are butterfly loops obtained by increasing the dissipation coefficient $\gamma_{1}^{(1)}$ whose value is listed in Table 1 (cases III and IV). The selected levels of stress are identical to those in Fig. 5. The dashed lines (case III in Table 1) in Fig. 6 neglecting the depolarization effect indicate that the actuation strains cannot be reduced by simply enlarging the dissipation coefficient $\gamma_{1}^{(1)}$ alone. On the other hand, the solid lines (case IV in Table 1) in Fig. 6 accounts for the depolarization effect, showing that the actuation strains in this case are further reduced by increasing $\gamma_{1}^{(1)}$.

Finally, with the illustration of Figs. 5 and 6, the effect of depolarization field and non-identical dissipation coefficients in different levels of laminate is taken into account in the simulated butterfly loops of Fig. 4(b) under various compressive stresses identical to those applied in experiment. The numerical values used to generate Fig. 4(b) are the same of those listed in the case IV of Table 1, where the value of $\gamma_{1}^{(1)}$ is chosen to fit the measured actuation strain under the compressive stress 5.84 MPa. Surprisingly the predicted actuation strains for other levels of compressive stress $(2.86,3.49,4.50 \mathrm{MPa})$ in Fig. 4(b) also agree quite well with the measured strains, as shown in Fig. 4(a). However, the shapes of these simulated curves are different qualitatively in several respects; in particular, the onset of $90^{\circ}$ switching occurs much earlier in Fig. 4(a) than in Fig. 4(b). This could be explained by our in situ measurement of $E_{90^{\circ}}^{\mathrm{c}}$. We have observed that a small portion of $90^{\circ}$ domains was developed at an early stage of the electric loading. To account for it, we identify two field points, i.e. the onset point $E_{90^{\circ}}^{\mathrm{c}, \mathrm{o}}$ and the finish point $E_{90^{\circ}}^{\mathrm{c}, \mathrm{f}}$. According to the experimental measurements, they are $E_{90^{\circ}}^{\mathrm{c}, \mathrm{o}}=0.06 \mathrm{MVm}^{-1}$ and $E_{90^{\circ}}^{\mathrm{c}, \mathrm{f}}=0.26 \mathrm{MVm}^{-1}$. We further assume that the $90^{\circ}$ coercive field starts at $E_{90^{\circ}}^{\mathrm{c}, \mathrm{o}}$ and rapidly increases to $E_{90^{\circ}}^{\mathrm{c}, \mathrm{f}}$ linearly at a small value of $\mu_{2}$. The simulated butterfly curves including this consideration are shown in Fig. 4(c). Clearly, the inclusion of the onset/finish field points as well as the interpolation between these two will not affect the magnitudes of attainable actuation strains. It only influences the initiation of $90^{\circ}$ switching and the shape of hysteresis loops. As the present study concerns the factors causing strain reduction and the precise interpolation between the onset and finish field points is not available from experiment, the exact shapes of butterfly loops are not pursued here.

\subsection{Comparison with other experiment}

Recently, Burcsu et al. (2004) have performed an experimental investigation of electrostrictive behavior of $\mathrm{BaTiO}_{3}$ single crystals using the flat-plate configuration. Although measured with an entirely different loading setup to the present study, they also observed the reduction in strain actuation. For comparison, the maximum actuation strains produced by the present and Burcsu et al.'s $\mathrm{BaTiO}_{3}$ single crystals are shown in Fig. 7 in the form of largest strain hysteresis loops. The former exhibits the maximum strain around $0.45 \%$ under the 
a

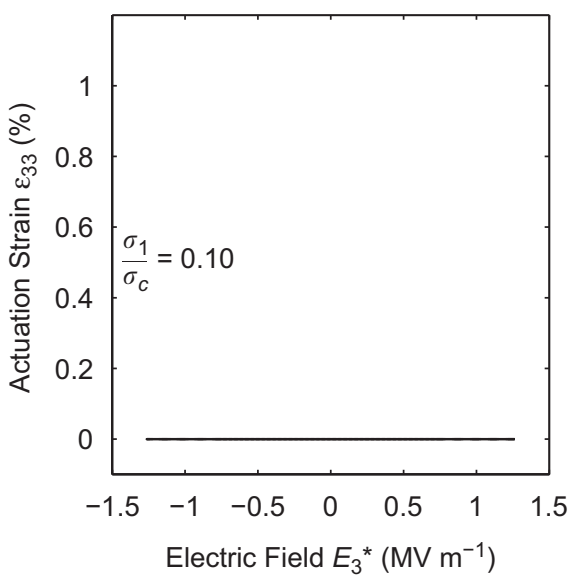

C

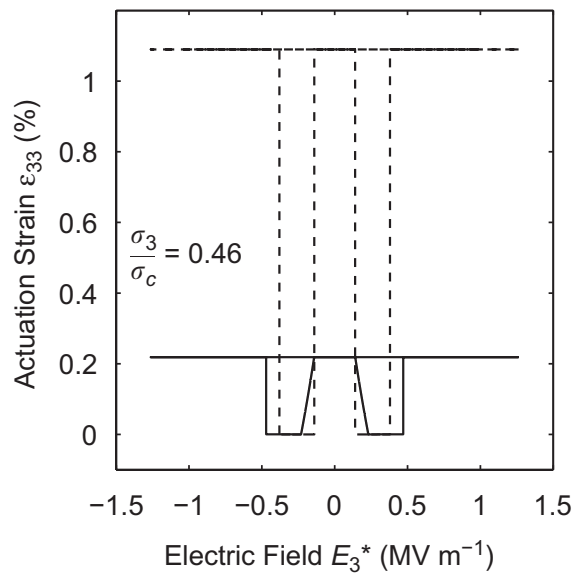

b

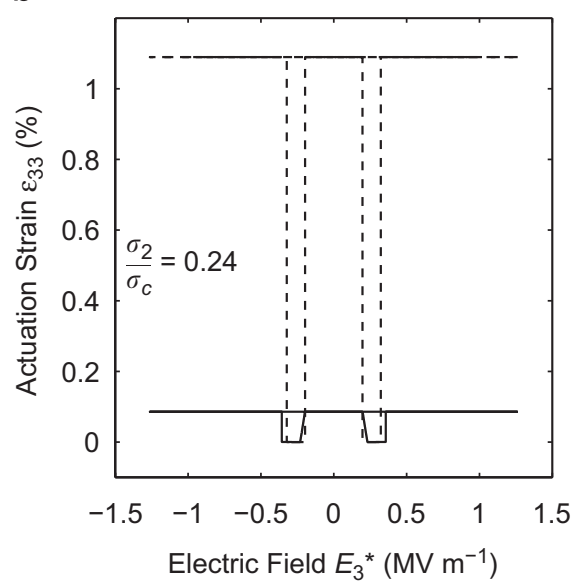

d

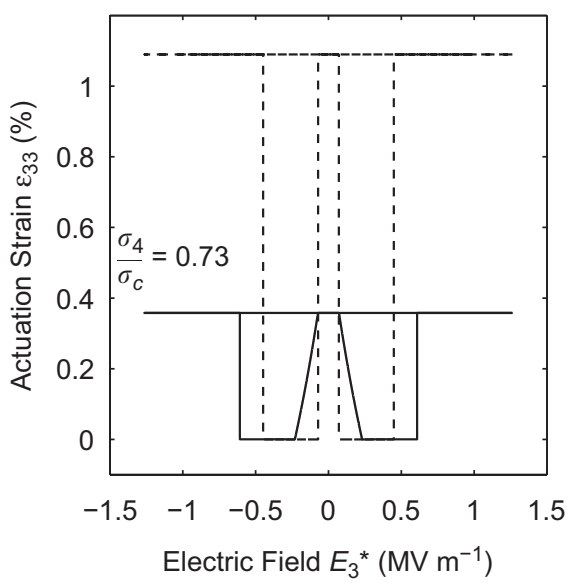

Fig. 6. Butterfly loops are simulated to demonstrate the effect of enlarging the dissipation coefficients in the lowest rank of laminate on the attainable actuation strains under various compressive stresses. The dashed/solid lines are the results excluding/including the depolarization effect, respectively. Note that $\sigma_{1}=0.6 \mathrm{MPa}, \sigma_{2}=1.5 \mathrm{MPa}, \sigma_{3}=2.86 \mathrm{MPa}, \sigma_{4}=4.5 \mathrm{MPa}$ and $\sigma^{\mathrm{c}}=6.2 \mathrm{MPa}$.

compressive stress of $5.84 \mathrm{MPa}$, while the latter shows a strain about $0.8 \%$ at $1.78 \mathrm{MPa}$ compressive stress. To simulate the wide difference observed in these two experiments, consider the switching criterion proposed by Eq. (22). The simulated strain hysteresis curve of the present crystal is taken from that shown in Fig. 4(c) at the maximum stress. To simulate the hysteresis behavior of Burcsu et al.'s crystal, however, only one of the coercive fields $E_{180^{\circ}}^{\mathrm{c}}=0.04 \mathrm{MVm}^{-1}$ has been measured. Another coercive field $E_{90^{\circ}}^{\mathrm{c}}$ is not available from their work. Thus, the data $E_{90^{\circ}}^{\mathrm{c}}=0.046 \mathrm{MVm}^{-1}$ measured by Li et al. (1992) is chosen, since the magnitudes of the switching stresses are similar in these two experimental works. Moreover, we take the same ratio $\gamma_{1}^{(1)} / 2 E_{180^{\circ}}^{\mathrm{c}} P_{\mathrm{s}}=3.5$ in these two simulations, and the results are also shown in Fig. 7 . It is obvious that the present switching model is capable of explaining the different electrostrictive behaviors observed by these two experiments.

The reason that the present crystal exhibits significantly lower strain outputs in comparison with Burcsu et al.'s crystal is explained via the argument of depolarization effect. From Fig. 2(a), the in-plane alternating layers of $180^{\circ}$ domains are formed to reduce the depolarization energy. But the domain pattern needs to remain compatible during evolution. As a result, under combined electromechanical loading, the development of local volume fraction $\mu_{1}$ induces both the $90^{\circ}$ and $180^{\circ}$ switchings. In other words, the presence of non-local electrostatic field requires concurrent $180^{\circ}$ switching, and therefore, the mobility of $180^{\circ}$ 


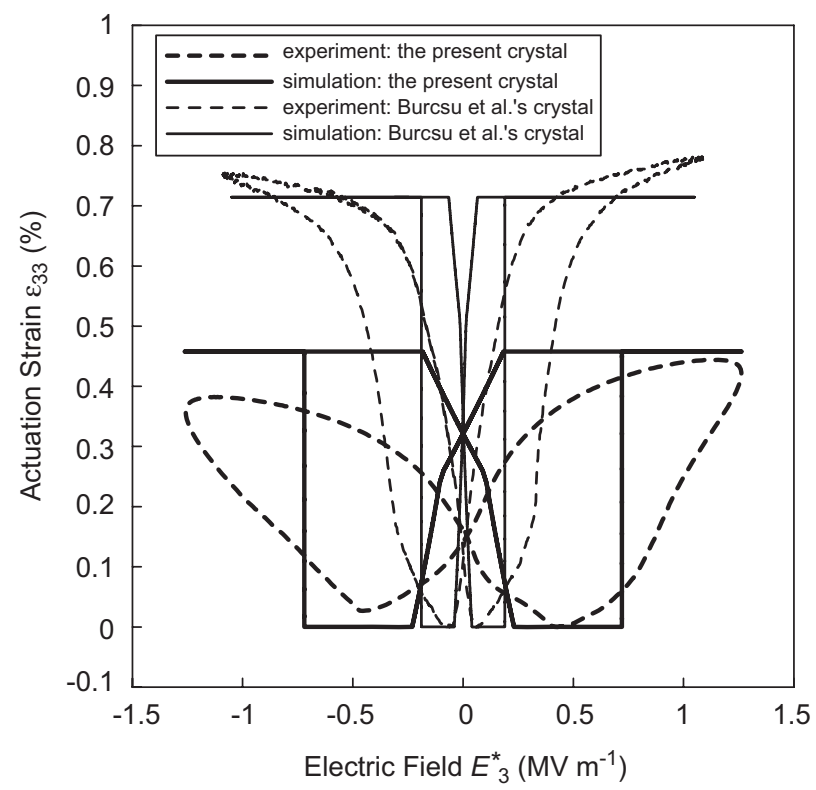

Fig. 7. Comparison of maximum actuation strains between the present and Burcsu et al.'s $\mathrm{BaTiO}_{3}$ single crystals (Burcsu et al., 2004) in the form of largest strain hysteresis loops. The simulated and measured butterfly hysteresis loops are presented using the thick solid and dashed lines for the present crystal, while using the thin solid and dashed lines for Burcsu et al.'s crystal.

domain walls may affect the electrostrictive hysteresis. Indeed, the measured coercive field $E_{180^{\circ}}^{\mathrm{c}}$ of the present crystal is about an order of magnitude larger than that of Burcsu et al.'s crystal. This may cause $90^{\circ}$ switching harder in the present crystal under combined electromechanical loading, giving rise to smaller strain in actuation.

\section{Conclusions}

In this article, a switching model is developed to predict the electromechanical behavior of a ferroelectric single crystal under applied electric field and stress. The framework starts with an energetic description of a ferroelectric crystal together with a treatment on the depolarization energy. It employs a domain configuration constructed by compatible multirank lamination, and that switching is assumed to be enabled by its evolution under loading. In addition, introducing laminated domains offers a great advantage of specifying different types of wall movements, leading to an explicit expression of critical resistance forces as in Eq. (24) or (27). All these features make the present framework a valuable alternative to the other celebrated switching models, such as the one developed by Huber et al. (1999).

The model is applied to explain the significant reduction in strain actuation observed in our recent experiment on $\mathrm{BaTiO}_{3}$ single crystals under the flat-plate configuration. Simulations based on two measured parameters $E_{180^{\circ}}^{\mathrm{c}}$ and $E_{90^{\circ}}^{\mathrm{c}}$ indicate strain reduction due to the inclusion of depolarization effect, as shown in Fig. 5. Other possible mechanisms such as friction effect and coercive hardening could also be adopted to explain it; however, they may need adjustable parameters. Moreover, the former is governed by the experimental setup and the latter is typically insignificant for single crystals.

Finally, the vastly different actuation strains produced by the present and Burcsu et al.'s $\mathrm{BaTiO}_{3}$ single crystals (Burcsu et al., 2004) are compared and explained using the proposed model. The simulated results shown in Fig. 7 are found in good agreement with the observed strains for these two different experiments. It confirms that the present framework is able to capture many important features in the electrostrictive behavior of ferroelectric single crystals, and thus is expected to assist the development of reliable constitutive models for ferroelectric polycrystals. 


\section{Acknowledgments}

We are grateful to K. Bhattacharya for many helpful discussions and to E. Burcsu for providing the experimental data shown in Fig. 7. The authors are glad to acknowledge the partial supports under Grant nos. NSC 96-2628-E-002-119-MY3, NSC 96-2221-E-002-159 and 96-EC-17-A-05-S1-017.

\section{Appendix A. Derivation of the depolarization coefficient $K$ in Eq. (19)}

Consider a ferroelectric crystal occupying the domain $\Omega=\left\{-L / 2<x_{1}<L / 2,-H / 2<x_{3}<H / 2\right\}$, where $L$ and $H$ denote the width and thickness of the rectangle. The electrodes are produced on the top and bottom edges. Let $\mathbf{p}=\left(p_{1}, p_{3}\right)$ be the average polarization inside the crystal. We assume that the system is completely shielded for $x_{3}>H / 2$ and $x_{3}<-H / 2$. The electric potential of the system can be obtained by solving the Maxwell's equation

$$
\begin{aligned}
& \frac{\partial^{2} \phi^{-}}{\partial x_{1}^{2}}+\frac{\partial^{2} \phi^{-}}{\partial x_{3}^{2}}=0, \quad-\frac{L}{2}<x_{1}<\frac{L}{2}, \quad-\frac{H}{2}<x_{3}<\frac{H}{2}, \\
& \frac{\partial^{2} \phi^{+}}{\partial x_{1}^{2}}+\frac{\partial^{2} \phi^{+}}{\partial x_{3}^{2}}=0, \quad x_{1}<-\frac{L}{2}, \quad x_{1}>\frac{L}{2}, \quad-\frac{H}{2}<x_{3}<\frac{H}{2},
\end{aligned}
$$

with boundary conditions given by

$$
\begin{aligned}
& \phi^{+}\left(x_{1}, \pm \frac{H}{2}\right)=0, \quad \phi^{-}\left(x_{1}, \pm \frac{H}{2}\right)=0 \\
& \phi^{+}\left( \pm \frac{L}{2}, x_{3}\right)=\phi^{-}\left( \pm \frac{L}{2}, x_{3}\right), \\
& -\varepsilon_{0} \frac{\partial \phi^{+}}{\partial x_{1}}\left( \pm \frac{L}{2}, x_{3}\right)+\varepsilon_{0} \frac{\partial \phi^{-}}{\partial x_{1}}\left( \pm \frac{L}{2}, x_{3}\right)=p_{1}, \\
& \phi^{+} \text {bounded as } x_{1} \rightarrow \pm \infty .
\end{aligned}
$$

As the solution of Eq. (A.1) together with Eq. (A.2) can be obtained following the standard technique of separation of variables, the depolarization energy density $w^{\mathrm{d}}$ can be shown to be

$$
\begin{aligned}
w^{\mathrm{d}}=\frac{W^{\mathrm{d}}}{H L} & =\frac{1}{2 H L} \int_{\Omega} \mathbf{p} \cdot \nabla \phi^{-} \mathrm{d} x_{1} \mathrm{~d} x_{3} \\
& =\frac{1}{2 \varepsilon_{0}}\left[8 \frac{H}{L} \sum_{n=1}^{\infty} \frac{\left[1-(-1)^{n}\right]}{n^{3} \pi^{3}}\left(1-\mathrm{e}^{-n \pi L / H}\right)\right] p_{1}^{2} .
\end{aligned}
$$

This in turn gives the depolarization coefficient $K$ by

$$
8 \frac{H}{L} \sum_{n=1}^{\infty} \frac{\left[1-(-1)^{n}\right]}{n^{3} \pi^{3}}\left(1-\mathrm{e}^{-n \pi L / H}\right) .
$$

However, Eq. (A.3) is viewed as an approximation since the assumption of completely electrical shielding for $x_{3}>H / 2$ and $x_{3}<-H / 2$ may not hold if $H / L$ is not very small.

\section{References}

Arlt, G., Sasko, P., 1980. Domain configuration and equilibrium size of domains in $\mathrm{BaTiO}_{3}$ ceramics. J. Appl. Phys. 51, 4956-4960. Bassiouny, E., Ghaleb, A.F., Maugin, G.A., 1988a. Thermodynamic relations for coupled electromechanical hysteresis effects I. Basic equations. Int. J. Eng. Sci. 26, 1279-1295. 
Bassiouny, E., Ghaleb, A.F., Maugin, G.A., 1988b. Thermodynamic relations for coupled electromechanical hysteresis effects II. Poling of ceramics. Int. J. Eng. Sci. 26, 1297-1306.

Bhattacharya, K., Ravichandran, G., 2003. Ferroelectric perovskites for electromechanical actuation. Acta Mater. 51, 5941-5960.

Bhattacharya, K., Li, J.Y., Shu, Y.C., 2008. Homogenization of Ferroelectric Polycrystals, in manuscript.

Burcsu, E., Ravichandran, G., Bhattacharya, K., 2000. Large strain electrostrictive actuation in barium titanate. Appl. Phys. Lett. 77, 1698-1700.

Burcsu, E., Ravichandran, G., Bhattacharya, K., 2004. Large electrostrictive actuation of barium titanate single crystals. J. Mech. Phys. Solids 52, 823-846.

Cao, W.W., 2005. The strain limits on switching. Nat. Mater. 4, 727-728.

Chen, L.Q., 2002. Phase-field models for microstructure evolution. Annu. Rev. Mater. Res. 32, 113-140.

Chen, X., Fang, D.N., Hwang, K.C., 1997. Micromechanics simulation of ferroelectric polarization switching. Acta Mater. 45, 3181-3189.

Dayal, K., Bhattacharya, K., 2007. A real-space non-local phase-field model of ferroelectric domain patterns in complex geometries. Acta Mater. 55, 1907-1917.

DeSimone, A., 1993. Energy minimizers for large ferromagnetic bodies. Arch. Ration. Mech. Anal. 125, $99-143$.

DeSimone, A., James, R.D., 2002. A constrained theory of magnetoelasticity. J. Mech. Phys. Solids, 283-320.

Elhadrouz, M., Zineb, T.B., Patoor, E., 2005. Constitutive law for ferroelectric and ferroelastic single crystals: a micromechanical approach. Comput. Mater. Sci. 32, 355-359.

Fotinich, Y., Carman, G.P., 2000. Stresses in piezoceramics undergoing polarization switchings. J. Appl. Phys. 88, 6715-6725.

Fulton, C.C., Gao, H., 2001. Microstructure modeling of ferroelectric fracture. Acta Mater. 49, $2039-2054$.

Hong, S., Colla, E.L., Kim, E., Taylor, D.V., Tagantsev, A.K., Muralt, P., No, K., Setter, N., 1999. High resolution study of domain nucleation and growth during polarization switching in $\mathrm{Pb}(\mathrm{Zr}, \mathrm{Ti}) \mathrm{O}_{3}$ ferroelectric thin film capacitors. J. Appl. Phys. 86, 607-613.

Huber, J.E., 2005. Micromechanical modelling of ferroelectrics. Curr. Opin. Solid State Mater. Sci. 9, 100-106.

Huber, J.E., Fleck, N.A., 2001. Multi-axial electrical switching of a ferroelectric: theory versus experiment. J. Mech. Phys. Solids 49, $785-811$.

Huber, J.E., Fleck, N.A., Landis, C.M., McMeeking, R.M., 1999. A constitutive model for ferroelectric polycrystals. J. Mech. Phys. Solids 47, 1663-1697.

Hwang, S.C., Lynch, C.S., McMeeking, R.M., 1995. Ferroelectric/ferroelastic interactions and a polarization switching model. Acta Metall. Mater. 43, 2073-2084.

James, R.D., 1986. Displacive phase transformations in solids. J. Mech. Phys. Solids 34, 359-394.

Jona, F., Shirane, G., 1962. Ferroelectric Crystals. Pergamon Press, New York.

Kamlah, M., 2001. Ferroelectric and ferroelastic piezoceramics - modeling of electromechanical hysteresis phenomena. Continuum Mech. Thermodyn. 13, 219-268.

Kamlah, M., Tsakmakis, C., 1999. Phenomenological modeling of the non-linear electromechanical coupling in ferroelectrics. Int. J. Solids Struct. 36, 669-695.

Kamlah, M., Liskowsky, A.C., McMeeking, R.M., Balke, H., 2005. Finite element simulation of a polycrystalline ferroelectric based on a multidomain single crystal switching model. Int. J. Solids Struct. 42, 2949-2964.

Kessler, H., Balke, H., 2001. On the local and average energy release in polarization switching phenomena. J. Mech. Phys. Solids 49 , 953-978.

Kim, S.J., Jiang, Q., 2002. A finite element model for rate-dependent behavior of ferroelectric ceramics. Int. J. Solids Struct. 39, 1015-1030.

Kim, S.J., Seelecke, S., 2007. A rate-dependent three-dimensional free energy model for ferroelectric single crystals. Int. J. Solids Struct. 44, 1196-1209.

Landis, C.M., 2002. Fully Coupled, multi-axial, symmetric constitutive laws for polycrystalline ferroelectric ceramics. J. Mech. Phys. Solids $50,127-152$.

Landis, C.M., 2004. Non-linear constitutive modeling of ferroelectrics. Curr. Opin. Solid State Mater. Sci. 8, 59-69.

Landis, C.M., McMeeking, R.M., 2001. A self-consistent constitutive model for switching in polycrystalline barium titanate. Ferroelectrics 255, 13-34.

Li, J.Y., Liu, D., 2004. On ferroelectric crystals with engineered domain configurations. J. Mech. Phys. Solids 52, $1719-1742$.

Li, W.F., Weng, G.J., 2002. A theory of ferroelectric hysteresis with a superimposed stress. J. Appl. Phys. 91, 3806-3815.

Li, W.F., Weng, G.J., 2004. A micromechanics-based thermodynamic model for the domain switch in ferroelectric crystals. Acta Mater. $52,2489-2496$.

Li, Z., Chan, S.K., Grimsditch, M.H., Zouboulis, E.S., 1991. The elastic and electromechanical properties of tetragonal BaTiO 3 single crystals. J. Appl. Phys. 70, 7327-7332.

Li, Z., Foster, C.M., Dai, X.H., Xu, X.Z., Chan, S.K., Lam, D.J., 1992. Piezoelectrically-induced switching of $90^{\circ}$ domains in tetragonal $\mathrm{BaTiO}_{3}$ and $\mathrm{PbTiO}_{3}$ investigated by micro-Raman spectroscopy. J. Appl. Phys. 71, 4481-4486.

Little, E.A., 1955. Dynamical behavior of domain walls in barium titanate. Phys. Rev. 98, 978-984.

Liu, T., Lynch, C.S., 2003. Ferroelectric properties of [ $\left.\begin{array}{llll}1 & 1 & 0\end{array}\right],\left[\begin{array}{lll}0 & 0 & 1\end{array}\right]$ and $\left[\begin{array}{lll}1 & 1 & 1\end{array}\right]$ poled relaxor single crystals: measurements and modeling. Acta Mater. 51, 407-416.

Loge, R.E., Suo, Z., 1996. Nonequilibrium thermodynamics of ferroelectric domain evolution. Acta Mater. 44, $3429-3438$.

Lu, W., Fang, D.N., Li, C.Q., Hwang, K.C., 1999. Nonlinear electric-mechanical behavior and micromechanics modelling of ferroelectric domain evolution. Acta Mater. 47, 2913-2926. 
Ma, Y.F., Li, J.Y., 2007. A constrained theory on actuation strain in ferromagnetic shape memory alloys induced by domain switching. Acta Mater. 55, 3261-3269.

McMeeking, R.M., Landis, C.M., 2002. A phenomenological multi-axial constitutive law for switching in polycrystalline ferroelectric ceramics. Int. J. Eng. Sci. 40, 1553-1577.

Michelitsch, T., Kreher, W.S., 1998. A simple model for the nonlinear material behavior of ferroelectrics. Acta Mater. 46, 5085-5094.

Park, S.E., Shrout, T.R., 1997. Ultrahigh strain and piezoelectric behavior in relaxor based ferroelectric single crystals. J. Appl. Phys. 82, 1804-1811.

Ren, X.B., 2004. Large electric-field-induced strain in ferroelectric crystals by Poin-defect-mediated reversible domain switching. Nat. Mater. 3, 91-94.

Ricote, J., Whatmore, R.W., Barber, D.J., 2000. Studies of the ferroelectric domain configuration and polarization of rhombohedral PZT ceramics. J. Phys. Condens. Matter 12, 323-337.

Rödel, J., Kreher, W.S., 2000. Self-consistent modelling of non-linear effective properties of polycrystalline ferroelectric ceramics. Comput. Mater. Sci. 19, 123-132.

Rödel, J., Kreher, W.S., 2003. Modelling linear and nonlinear behavior of polycrystalline ferroelectric ceramics. J. Eur. Ceramic Soc. 23, 2297-2306.

Seelecke, S., Kim, S.J., Ball, B.L., Smith, R.C., 2005. A rate-dependent two-dimensional free energy model for ferroelectric single crystals. Continuum Mech. Thermodyn. 17, 337-350.

Shieh, J., Shu, Y.C., 2008. Polarization-free strain changes in single crystal and polycrystalline ferroelectrics, in preparation.

Shieh, J., Huber, J.E., Fleck, N.A., Ashby, M.F., 2001. The selection of sensors. Prog. Mater. Sci. 46, 461-504.

Shieh, J., Huber, J.E., Fleck, N.A., 2003. An evaluation of switching criteria for ferroelectrics under stress and electric field. Acta Mater. 51, 6123-6137.

Shieh, J., Yeh, J.H., Shu, Y.C., Yen, J.H., 2007. Operation of multiple $90^{\circ}$ switching systems in barium titanate single crystals under electromechanical loading. Appl. Phys. Lett. 91, 062901.

Shilo, D., Burcsu, E., Ravichandran, G., Bhattacharya, K., 2007. A model for large electrostrictive actuation in ferroelectric single crystals. Int. J. Solids Struct. 44, 2053-2065.

Shu, Y.C., Bhattacharya, K., 2001. Domain patterns and macroscopic behavior of ferroelectric materials. Philos. Mag. B 81, $2021-2054$.

Shu, Y.C., Yen, J.H., 2007. Pattern formation in martensitic thin films. Appl. Phys. Lett. 91, 021908.

Shu, Y.C., Lin, M.P., Wu, K.C., 2004. Micromagnetic modeling of magnetostrictive materials under intrinsic stress. Mech. Mater. 36, 975-997.

Shu, Y.C., Yen, J.H., Chen, H.Z., Li, J.Y., Li, L.J., 2008. Constrained modeling of domain patterns in rhombohedral ferroelectrics. Appl. Phys. Lett. 92, 052909.

Shu, Y.C., Yen, J.H., Shieh, J., Yeh, J.H., 2007. Effect of depolarization and coercivity on actuation strains due to domain switching in barium titanate. Appl. Phys. Lett. 90, 172902.

Smith, R.C., Seelecke, S., Dapino, M.J., Ounaies, Z., 2005. A unified framework for modeling hysteresis in ferroic materials. J. Mech. Phys. Solids 54, 46-85.

Sun, C.T., Achuthan, A., 2004. Domain-switching criteria for ferroelectric materials subjected to electrical and mechanical loads. J. Am. Ceramic Soc. 87, 395-400.

Uchino, K., 1998. Materials issues in design and performance of piezoelectric actuators: an overview. Acta Mater. 46, $3745-3753$.

Wada, S., Suzuki, S., Noma, T., Suzuki, T., Osada, M., Kakihana, M., Park, S.E., Cross, L.E., Shrout, T.R., 1999. Enhanced piezoelectric property of barium titanate single crystals with engineered domain configurations. Jpn. J. Appl. Phys. 38, 5505-5511.

Yen, J.H., 2008. Ph.D. Thesis. National Taiwan University, in preparation.

Zhang, W., Bhattacharya, K., 2005a. A computational model of ferroelectric domains. Part I: model formulation and domain switching. Acta Mater. 53, 185-198.

Zhang, W., Bhattacharya, K., 2005b. A computational model of ferroelectric domains. Part II: grain boundaries and defect pinning. Acta Mater. 53, 199-209.

Zhang, Z.K., Fang, D.N., Soh, A.K., 2006. A new criterion for domain-switching in ferroelectric materials. Mech. Mater. 38, 25-32. 